\title{
EXTENSION OF THE PAST LIFETIME AND ITS CONNECTION TO THE CUMULATIVE ENTROPY
}

\author{
ANTONIO DI CRESCENZO, ${ }^{*}$ Università degli Studi di Salerno \\ ABDOLSAEED TOOMAJ, ${ }^{* *}$ Gonbad Kavous University
}

\begin{abstract}
Given two absolutely continuous nonnegative independent random variables, we define the reversed relevation transform as dual to the relevation transform. We first apply such transforms to the lifetimes of the components of parallel and series systems under suitably proportionality assumptions on the hazard rates. Furthermore, we prove that the (reversed) relevation transform is commutative if and only if the proportional (reversed) hazard rate model holds. By repeated application of the reversed relevation transform we construct a decreasing sequence of random variables which leads to new weighted probability densities. We obtain various relations involving ageing notions and stochastic orders. We also exploit the connection of such a sequence to the cumulative entropy and to an operator that is dual to the Dickson-Hipp operator. Iterative formulae for computing the mean and the cumulative entropy of the random variables of the sequence are finally investigated.
\end{abstract}

Keywords: Relevation transform; reversed relevation transform; proportional hazard rate model; proportional reversed hazard rate model; weighted cumulative distribution; cumulative entropy

2010 Mathematics Subject Classification: Primary 60E15

Secondary 62B $10 ; 62 \mathrm{~N} 05 ; 94 \mathrm{~A} 17$

\section{Introduction}

In this paper we introduce the reversed relevation transform and study some properties of a new weighted cumulative distribution function and its connection with the cumulative entropy. The considered stochastic model is dual to the weighted tail distribution studied by Kapodistria and Psarrakos [18]. Specifically, we construct a sequence of stochastically decreasing random variables $\left\{X_{n}, n \geq 1\right\}$. In this sequence, $X_{1}$ is nonnegative and absolutely continuous, and the $(n+1)$ th random variable of the sequence is inductively defined through the following relation: $\left[X_{n+1} \mid X_{n}=t\right] \stackrel{\mathrm{D}}{=}\left[X_{n} \mid X_{n} \leq t\right], n=1,2, \ldots$, for $t>0$. Here, as usual, $[X \mid B]$ denotes a random variable having the same distribution of $X$ conditional on $B$, and ' $=$, denotes equality in distribution.

Roughly speaking, $\left\{X_{n}, n \geq 1\right\}$ is suitable to describe an iterative process involving a sequence of tasks, where $X_{n}$ is the random time required to perform the $n$th task. For instance, we refer to a training procedure based on iterative learning or a working system based on replacements or repairs of failed items. Starting from the sequence $\left\{X_{n}, n \geq 1\right\}$, we derive some

\footnotetext{
Received 17 July 2014; revision received 23 December 2014.

* Postal address: Dipartimento di Matematica, Università degli Studi di Salerno, Via Giovanni Paolo II n.132, I-84084

Fisciano (SA), Italy. Email address: adicrescenzo@unisa.it

** Postal address: Department of Statistics, Gonbad Kavous University, Gonbad Kavous, Iran.

Email address: ab.toomaj@gmail.com
} 
properties of a new weighted cumulative distribution via stochastic orders, and its connection with covariance and cumulative entropy. Our investigation is also devoted to disclosing iterative rules that allow us to compute the mean and the cumulative entropy of the random variables of the sequence, whose computational efficiency is illustrated by some numerical examples.

We recall that [18] constructed a stochastically increasing sequence of random variables, whose iterative rule involved the residual lifetime of $X_{n}$, i.e.

$$
\left[X_{n+1}-X_{n} \mid X_{n}=t\right] \stackrel{\mathrm{D}}{=}\left[X_{n}-t \mid X_{n}>t\right], \quad n \geq 1, t>0 .
$$

They obtained some results on this sequence and its connections to the cumulative residual entropy. Their process may describe the successive failures of a component, which, on failure, is replaced by a component of equal age, but the lifetime distribution of the $n$th component is assumed to be identical to the distribution of the time until the $n$th failure; see [18] for more details. A simpler case was studied by Baxter [3], who considered a stochastic process generated by the successive failures of a component which on failure is replaced by a component of equal age.

This paper is organized as follows. In Section 2 we present the reversed relevation transform with a preliminary result based on the usual stochastic order and an application to parallel systems involving the proportional reversed hazard rate model (PRHRM). Some dual results for the relevation transform are then provided. We also address the problem of determining necessary and sufficient conditions such that the reversed relevation transform and the relevation transform are commutative. The new weighted distributions and their characteristics based on stochastic orders and ageing properties are discussed in Section 3, where the new notion of decreasing reversed hazard rate (DRHR) in a length-biased sense is also considered. In Section 4 various kinds of entropy such as Shannon entropy, cumulative entropy, and dynamic cumulative entropy are examined. Then the connections between the earlier mentioned entropies and several functions of the given sequence are discussed. Specifically, we also obtain some iterative results for the involved quantities. These include a new probabilistic meaning for the cumulative entropy, which can be expressed as a difference of means of consecutive random variables of the considered sequence. Finally, in Section 5 we define an integral operator and we discuss its properties related to the previous results. Also, various numerical examples are presented to shed further light on the characteristics of the studied sequence.

\section{Background and preliminary results}

Let $X$ be an absolutely continuous nonnegative random variable with probability density function (PDF) $f(t)$, cumulative distribution function (CDF) $F(t)=\mathbb{P}(X \leq t)$, and survival function $\bar{F}(t)=1-F(t)$, so that $X$ may be viewed as the random lifetime of a system or a component or a living organism. Assume that $F(t)>0$ for all $t>0$. We recall that the reversed hazard rate function of $X$ is defined by

$$
\tau(t)=\frac{\mathrm{d}}{\mathrm{d} t} \log F(t)=\frac{f(t)}{F(t)}, \quad t>0
$$

There are several papers on applications of the reversed hazard rate function in the literature; see, e.g. Block et al. [4], Di Crescenzo [10], Gupta and Nanda [17], Kijima and Ohnishi [19], and the references therein. For the random lifetime $X$, we define $X_{[t]}=[t-X \mid X \leq t]$, $t>0$, which is termed the inactivity time. Indeed, $X_{[t]}$ describes the length of the time interval occurring between the failure time $X$ and an inspection time $t$, given that at time $t$ the system 
has been found failed. For $t>0$, the mean inactivity time of $X$ is given by

$$
\tilde{\mu}(t)=\mathbb{E}[t-X \mid X \leq t]=\frac{1}{F(t)} \int_{0}^{t} F(x) \mathrm{d} x .
$$

It is known that the CDF of the past lifetime $[X \mid X \leq t], t>0$, is given by

$$
\mathbb{P}(X \leq x \mid X \leq t)= \begin{cases}\frac{F(x)}{F(t)}, & 0 \leq x \leq t, \\ 1, & x>t,\end{cases}
$$

so that the PDF of the past lifetime is $f(x) / F(t)$ for all $0<x<t$.

Hereafter, we consider two nonnegative absolutely continuous and independent random variables $X$ and $Y$ with the CDFs $F(t)$ and $G(t)$, respectively.

Definition 1. The reversed relevation transform of $X$ and $Y$ is defined by

$$
\begin{aligned}
G \tilde{\#} F(x) & =\int_{0}^{\infty}\left\{\frac{F(x)}{F(t)} \mathbf{1}_{\{0 \leq x \leq t\}}+\mathbf{1}_{\{x>t\}}\right\} \mathrm{d} G(t) \\
& =G(x)+F(x) \int_{x}^{\infty} \frac{1}{F(t)} \mathrm{d} G(t), \quad x>0,
\end{aligned}
$$

where $\mathbf{1}_{A}$ is the indicator function of the set $A$, i.e. $\mathbf{1}_{A}(x)=1$ if $x \in A$, and $\mathbf{1}_{A}(x)=0$ if $x \in A^{c}$.

Generally, the inactivity time of $X$ at a random time $Y$, denoted by $X_{[Y]}$, is defined as $X_{[Y]} \stackrel{\mathrm{D}}{=}[Y-X \mid X \leq Y]$; see, e.g. [18]. Moreover, let $X[Y] \stackrel{\mathrm{D}}{=}[X \mid X \leq Y]$ denote the total time of $X$ given that it is less than an independent random inspection time $Y$. Therefore, the $\mathrm{CDF}$ of $X[Y]$ is given by

$$
\mathbb{P}(X[Y] \leq x)=G \tilde{\#} F(x),
$$

where the symbol $\widetilde{\#}$ is defined in (2). If random variables $X$ and $Y$ are independent and identically distributed (i.i.d.), then

$$
\mathbb{P}(X[Y] \leq x)=F \tilde{\#} F(x)=F(x)[1+T(x)], \quad x>0,
$$

where

$$
T(x)=-\log F(x)=\int_{x}^{\infty} \tau(u) \mathrm{d} u, \quad x>0,
$$

denotes the cumulative reversed hazard rate function of $X$; see, e.g. [10].

Example 1. (a) Let $X$ and $Y$ be independent nonnegative random variables having CDFs $F(x)=\exp \left(-a x^{-\gamma}\right), x>0$, and $G(x)=\exp \left(-c x^{-\gamma}\right), x>0$, respectively, with $a, c, \gamma>0$. From (2) and (3), we have

$$
\mathbb{P}(X[Y] \leq x)=G \# F(x)= \begin{cases}\frac{1}{c-a}\left(c \exp \left(-a x^{-\gamma}\right)-a \exp \left(-c x^{-\gamma}\right)\right), & x>0, a \neq c, \\ \left(1+c x^{-\gamma}\right) \exp \left(-c x^{-\gamma}\right), & x>0, a=c .\end{cases}
$$


(b) If $F(x)=\mathrm{e}^{-a /\left(\mathrm{e}^{x}-1\right)}, x>0$, and $G(x)=\mathrm{e}^{-c /\left(\mathrm{e}^{x}-1\right)}, x>0$, with $a, c>0$, then

$$
\mathbb{P}(X[Y] \leq x)=G \tilde{\#} F(x)= \begin{cases}\frac{1}{c-a}\left(c \mathrm{e}^{-a /\left(\mathrm{e}^{x}-1\right)}-a \mathrm{e}^{-c /\left(\mathrm{e}^{x}-1\right)}\right), & x>0, a \neq c, \\ \left(1+\frac{c}{\mathrm{e}^{x}-1}\right) \mathrm{e}^{-c /\left(\mathrm{e}^{x}-1\right)}, & x>0, a=c .\end{cases}
$$

Ageing notions and stochastic orders have many applications in various areas of science such as reliability and survival analysis, economics, insurance, actuarial and management sciences, and coding theory; see Shaked and Shanthikumar [32] for a detailed account. In the following, we review some notions that are used in the sequel. Note that here and throughout this paper, the terms 'increasing' and 'decreasing' are used in a nonstrict sense, and $\mathbb{R}$ denotes the set of real numbers.

Definition 2. If $X$ is an absolutely continuous random variable with support $\left(l_{X}, u_{X}\right), \mathrm{CDF} F$, PDF $f$, and reversed hazard rate function $\tau(t)=f(t) / F(t)$, then

- $X$ is said to have the increasing likelihood ratio (ILR) property if $f(x)$ is log-concave or, equivalently, the function $f^{\prime}(x) / f(x)$ is decreasing in $x \in\left(l_{X}, u_{X}\right)$;

- $X$ is said to have the decreasing likelihood ratio (DLR) property if $f(x)$ is log-convex or, equivalently, the function $f^{\prime}(x) / f(x)$ is increasing in $x \in\left(l_{X}, u_{X}\right)$;

- $X$ has the DRHR if $\tau(t)$ is decreasing in $t \in\left(l_{X}, u_{X}\right)$ or, equivalently, $T(x)=-\log F(x)$ is convex.

Moreover, if $Y$ is an absolutely continuous random variable with support $\left(l_{Y}, u_{Y}\right), \operatorname{CDF} G$, and PDF $g$, then

- $X$ is smaller than $Y$ in the usual stochastic order (denoted by $X \leq$ st $Y$ ) if $\bar{F}(t) \leq \bar{G}(t)$ for all $t \in \mathbb{R}$ or, equivalently, $F(t) \geq G(t)$ for all $t \in \mathbb{R}$;

- $X$ is smaller than $Y$ in the likelihood ratio order (denoted by $X \leq \operatorname{lr} Y$ ) if $f(x) g(y) \geq$ $f(y) g(x)$ for all $x \leq y$, with $x, y \in\left(l_{X}, u_{X}\right) \cup\left(l_{Y}, u_{Y}\right)$;

- $X$ is smaller than $Y$ in the up-shifted likelihood ratio order (denoted by $X \leq_{\operatorname{lr} \uparrow} Y$ ) if $X-x \leq \operatorname{lr} Y$ for all $x \geq 0$ or, equivalently, for each $x \geq 0$, we have $g(t) / f(t+x)$ is increasing in $t \in\left(l_{X}-x, u_{X}-x\right) \cup\left(l_{Y}, u_{Y}\right)$, where $a / 0$ is taken to be equal to $\infty$ whenever $a>0$.

Proposition 1. If $X$ and $Y$ are independent nonnegative random variables, then

$$
X[Y] \leq_{\mathrm{st}} \min \{X, Y\}
$$

Proof. Due to (2) and (3), for $x>0$, we have

$$
\begin{aligned}
\mathbb{P}(X[Y] \leq x) & =G(x)+F(x) \int_{x}^{\infty} \frac{1}{F(t)} \mathrm{d} G(t) \\
& \geq G(x)+F(x) \int_{x}^{\infty} \mathrm{d} G(t) \\
& =G(x)+F(x)-F(x) G(x) .
\end{aligned}
$$

The proof thus follows recalling that the last term is the $\operatorname{CDF}$ of $\min \{X, Y\}$. 
Let us now consider a stochastic model that extends both cases treated in Example 1. Let $X$ and $Y$ be absolutely continuous nonnegative random variables with CDFs $F(x)$ and $G(x)$, and reversed hazard rate functions $\tau_{X}(x)$ and $\tau_{Y}(x)$, respectively. These variables satisfy the PRHRM with proportionality constant $\theta>0$ if $\tau_{Y}(x)=\theta \tau_{X}(x)$ for all $x>0$ or, equivalently, if

$$
G(x)=[F(x)]^{\theta}, \quad x>0 .
$$

The parent distribution function can be expressed as $F(x)=\mathrm{e}^{-T(x)}, x>0$, where $T(x)$ is defined in (4). The model (5) was first proposed by Lehman [23] in contrast to the proportional hazard rate model. It is more flexible to accommodate both monotonic as well as nonmonotonic failure rates even though the baseline failure rate is monotonic. For more details on the applications and properties of the proportional hazard rate model see, e.g. [10], [15], [16], [26], and [27], among others.

Proposition 2. Under the PRHRM (5), we have

$$
G \tilde{\#} F(x)=\frac{1}{\theta-1}\left(\theta \mathrm{e}^{-T(x)}-\mathrm{e}^{-\theta T(x)}\right), \quad x>0,
$$

for $\theta>0, \theta \neq 1$.

Proof. Under the PRHRM (5), we can verify that

$$
\int_{x}^{\infty} \frac{1}{F(t)} \mathrm{d} G(t)=\frac{\theta}{\theta-1}\left(1-\mathrm{e}^{-(\theta-1) T(x)}\right), \quad x>0 .
$$

This identity and (2) thus yield (6).

Note that the assumptions of Proposition 2 are satisfied by the cases (a) and (b) shown in Example 1, for $a \neq c$.

Example 2. Let $X_{m: m}=\max \left\{X_{1}, \ldots, X_{m}\right\}$ be the lifetime of the parallel system consisting of $m$ components with absolutely continuous i.i.d. lifetimes $X_{1}, \ldots, X_{m}$ having the common CDF $F$. Moreover, suppose that $X_{i}, i=1, \ldots, m$, and $Y$ satisfy the PRHRM with proportionality constant $\theta$, as in (5). Then after some calculations, from (2) we can express the CDF of $X_{m: m}[Y]$ as the following generalized mixture:

$$
G \tilde{\#} F_{m: m}(x)=\frac{\theta F^{m}(x)-m F^{\theta}(x)}{\theta-m}, \quad x>0,
$$

for $\theta \neq m$.

In the following theorem we investigate the commutative property for the reversed relevation transform.

Theorem 1. The reversed relevation transform of $X$ and $Y$ is commutative if and only if $X$ and $Y$ satisfy the PRHRM.

Proof. If $X$ and $Y$ satisfy the PRHRM as specified in (5), then (2) yields

$$
G \widetilde{\#} F(x)=F \widetilde{\#} G(x)=\frac{\theta F(x)-G(x)}{\theta-1}, \quad x>0,
$$


for $\theta>0, \theta \neq 1$, and the desired result follows. To prove the converse, we assume that, for all $x>0$,

$$
G(x)+F(x) \int_{x}^{\infty} \frac{1}{F(t)} \mathrm{d} G(t)=F(x)+G(x) \int_{x}^{\infty} \frac{1}{G(t)} \mathrm{d} F(t) .
$$

Differentiating both sides of (7), we have

$$
f(x) \int_{x}^{\infty} \frac{1}{F(t)} \mathrm{d} G(t)=g(x) \int_{x}^{\infty} \frac{1}{G(t)} \mathrm{d} F(t),
$$

where $f$ and $g$ denote the PDFs of $X$ and $Y$, respectively. Again, differentiating both sides of (8), we obtain

$$
f^{\prime}(x) \int_{x}^{\infty} \frac{1}{F(t)} \mathrm{d} G(t)-\frac{f(x) g(x)}{F(x)}=g^{\prime}(x) \int_{x}^{\infty} \frac{1}{G(t)} \mathrm{d} F(t)-\frac{f(x) g(x)}{G(x)} .
$$

From (7)-(9) and some algebraic simplification, we obtain

$$
\frac{f^{\prime}(x)}{f(x)}-\frac{f(x)}{F(x)}=\frac{g^{\prime}(x)}{g(x)}-\frac{g(x)}{G(x)}, \quad x>0,
$$

i.e.

$$
\frac{\mathrm{d}}{\mathrm{d} x} \ln \frac{f(x)}{F(x)}=\frac{\mathrm{d}}{\mathrm{d} x} \ln \frac{g(x)}{G(x)}, \quad x>0 .
$$

Integration on both sides yields

$$
\ln \frac{f(x)}{F(x)}=\ln \frac{g(x)}{G(x)}+\text { constant, } \quad x>0,
$$

or

$$
\frac{f(x)}{F(x)}=\theta \frac{g(x)}{G(x)}, \quad x>0,
$$

where $\theta$ is a positive constant. Thus, we obtain $G(x)=[F(x)]^{\theta}, x>0$, which completes the proof.

Hereafter, we analyse some results that are dual to those given above. Let $X$ and $Y$ be absolutely continuous nonnegative random variables with survival functions $\bar{F}(x)$ and $\bar{G}(x)$, and hazard rate functions $h_{X}(x)=-(\mathrm{d} / \mathrm{d} x) \log \bar{F}(x)$ and $h_{Y}(x)=-(\mathrm{d} / \mathrm{d} x) \log \bar{G}(x)$, respectively. Then $X$ and $Y$ satisfy the proportional hazard rate model with proportionality constant $\theta>0$, if $h_{Y}(x)=\theta h_{X}(x)$ for all $x>0$. This is equivalent to the model

$$
\bar{G}(x)=[\bar{F}(x)]^{\theta}, \quad \theta>0,
$$

where $\bar{F}(x)=\mathrm{e}^{-\Lambda(x)}, x>0$, is the parent survival function and $\Lambda(x)=-\log \bar{F}(x)$ denotes the hazard function; see, e.g. [15]. Let $X(Y)$ denote the total time of $X$ given that it exceeds an independent random inspection time $Y$, i.e. $X(Y)=[X \mid X>Y]$. Then we have

$$
\mathbb{P}(X(Y)>x)=\bar{G} \# \bar{F}(x)=\bar{G}(x)+\bar{F}(x) \int_{0}^{x} \frac{1}{\bar{F}(t)} \mathrm{d} G(t), \quad x>0,
$$

where the symbol \# denotes the relevation transform introduced by Krakowski [20]. Equation (11) was discussed in [18]; see also [6] and [30] and the references therein. 
The following result is analogous to Proposition 1 and, thus, the proof is omitted.

Proposition 3. If $X$ and $Y$ are independent nonnegative random variables, then

$$
X(Y) \geq_{\text {st }} \max \{X, Y\} .
$$

Let us now see the analog of Proposition 2.

Proposition 4. Under the proportional hazard rate model (10), we have

$$
\bar{G} \# \bar{F}(x)=\frac{1}{\theta-1}\left(\theta \mathrm{e}^{-\Lambda(x)}-\mathrm{e}^{-\theta \Lambda(x)}\right), \quad x>0,
$$

for $\theta>0, \theta \neq 1$.

Proof. Since

$$
\int_{0}^{x} \frac{1}{\bar{F}(t)} \mathrm{d} G(t)=\frac{\theta}{\theta-1}\left(1-\mathrm{e}^{-(\theta-1) \Lambda(x)}\right), \quad x>0,
$$

the proof follows from (11).

Example 3. Suppose that $X_{1: m}=\min \left\{X_{1}, \ldots, X_{n}\right\}$ is the lifetime of the series system consisting of $m$ components with absolutely continuous i.i.d. lifetimes $X_{1}, \ldots, X_{m}$, having the common CDF $F$. Also, suppose that $X_{i}, i=1, \ldots, m$, and $Y$ satisfy the proportional hazard rate model with proportionality constant $\theta$, as in (10). By means of some calculations, from (11) we obtain the survival function of $X_{1: m}(Y)$, which is expressed as a generalized mixture:

$$
\bar{G} \# \bar{F}_{1: m}(x)=\frac{\theta \bar{F}^{m}(x)-m \bar{F}^{\theta}(x)}{\theta-m}, \quad x>0,
$$

for $\theta \neq m$.

The relevation transform is not always commutative. Indeed, in the following theorem we give a necessary and sufficient condition leading to such a property. Being similar to Theorem 1 we provide only a sketch of the proof.

Theorem 2. The relevation transform of $X$ and $Y$ is commutative if and only if $X$ and $Y$ satisfy the proportional hazard rate model.

Proof. Let $X$ and $Y$ satisfy the proportional hazard rate model as in (10). From (11), we thus have

$$
\bar{G} \# \bar{F}(x)=\bar{F} \# \bar{G}(x)=\frac{\theta \bar{F}(x)-\bar{G}(x)}{\theta-1}, \quad x>0,
$$

for $\theta>0, \theta \neq 1$, and then the relevation transform is commutative. To prove the converse, we assume that, for all $x>0$,

$$
\bar{G}(x)+\bar{F}(x) \int_{0}^{x} \frac{1}{\bar{F}(t)} \mathrm{d} G(t)=\bar{F}(x)+\bar{G}(x) \int_{0}^{x} \frac{1}{\bar{G}(t)} \mathrm{d} F(t) .
$$

Differentiating both sides and after some calculations, we obtain

$$
\frac{f^{\prime}(x)}{f(x)}+\frac{f(x)}{\bar{F}(x)}=\frac{g^{\prime}(x)}{g(x)}+\frac{g(x)}{\bar{G}(x)}, \quad x>0,
$$


so that

$$
\frac{\mathrm{d}}{\mathrm{d} x} \ln \frac{f(x)}{\bar{F}(x)}=\frac{\mathrm{d}}{\mathrm{d} x} \ln \frac{g(x)}{\bar{G}(x)}, \quad x>0 .
$$

Such a relation implies that $\bar{G}(x)=[\bar{F}(x)]^{\theta}, x>0$, for $\theta>0$, thus completing the proof.

The characterization of distributions based on the relevation transform has been the object of various investigations; see, e.g. Lau and Prakasa Rao [21] and [22]. We point out that $[14$, Theorem 9] states that the relevation transform of two i.i.d. nonnegative continuous random variables is identically distributed to their convolution, i.e. $F \# F(x)=F * F(x)$ for all $x \geq 0$, if and only if they have exponential distribution. However, due to Proposition 1, a similar result cannot hold for the reversed relevation transform.

\section{Sequence of weighted distributions}

Let $X$ be an absolutely continuous nonnegative random variable with PDF $f(x)$ and CDF $F(x)$. Based on $X$, we construct a sequence of random variables $\left\{X_{n}, n \geq 1\right\}$ as

$$
X_{1} \stackrel{\mathrm{D}}{=} X, \quad\left[X_{n+1} \mid X_{n}=t\right] \stackrel{\mathrm{D}}{=}\left[X_{n} \mid X_{n} \leq t\right], \quad n \geq 1
$$

or, equivalently,

$$
X_{1} \stackrel{\mathrm{D}}{=} X, \quad X_{n+1} \stackrel{\mathrm{D}}{=}\left[X_{n} \mid X_{n} \leq X_{n}^{\prime}\right], \quad n \geq 1,
$$

where $X_{n}^{\prime}$ is an independent copy of $X_{n}$. It is easy to show that the corresponding CDFs $F_{n}(x)=\mathbb{P}\left(X_{n} \leq x\right)$ are given as, for all $x>0$,

$$
F_{1}(x)=F(x), \quad F_{n+1}(x)=F_{n} \tilde{\#} F_{n}(x)=F_{n}(x)\left[1+T_{n}(x)\right], \quad n \geq 1,
$$

where

$$
T_{n}(x)=-\log F_{n}(x)=\int_{x}^{\infty} \tau_{n}(u) \mathrm{d} u, \quad x>0,
$$

denotes the cumulative hazard function of $X_{n}$, and

$$
\tau_{n}(u)=\frac{f_{n}(u)}{F_{n}(u)}, \quad u>0,
$$

is the reversed hazard rate of $X_{n}$. From (14), we can see that the corresponding densities are given by, for $x>0$,

$$
f_{1}(x)=f(x), \quad f_{n+1}(x)=T_{n}(x) f_{n}(x), \quad n \geq 1
$$

Due to (15), for $x>0$, we have

$$
T_{n+1}(x)=-\log F_{n+1}(x)=T_{n}(x)-\log \left(1+T_{n}(x)\right), \quad n \geq 1,
$$

and, thus, from (17), we obtain

$$
f_{n+1}(x)=T_{n}(x) f_{n}(x)=T_{n}(x) T_{n-1}(x) f_{n-1}(x)=\cdots=\prod_{i=1}^{n} T_{i}(x) f(x), \quad n \geq 1 .
$$


From (18), we see that $f_{n+1}(x)$ is a sequence of weighted PDFs. We recall that, given an absolutely continuous random variable $X$ having density $f$ and a nonnegative real function $w$, the associated weighted random variable $X^{w}$ has the PDF

$$
f^{w}(x)=\frac{w(x) f(x)}{\mathbb{E}[w(X)]}, \quad x \in \mathbb{R},
$$

provided that $0<\mathbb{E}[w(X)]<\infty$. See [25], [29], and [2] for some recent papers on weighted distributions.

The sequence of random variables $\left\{X_{n}, n \geq 1\right\}$ is suitable to describe an iterative process, where $X_{n}$ denotes the random time required to perform a task at the $n$th stage. For instance, consider a training procedure where, given that the $n$th learning time $X_{n}$ has duration $t$, the $(n+1)$ th random time is identically distributed to $X_{n}$ conditional on $X_{n} \leq t$. In some sense, (12) expresses that the information collected at each stage allows the next step of the procedure to have a stochastically smaller duration. Alternatively, $\left\{X_{n}, n \geq 1\right\}$ may be viewed as the sequence of lifetimes of an item that is repaired instantaneously after each failure, such that after each repair the duration of the next lifetime is stochastically smaller than the previous, due to imperfect repairs and weakening caused by wear.

From (14), since $T_{n}(x) \geq 0$ for all $x>0$ and $n \geq 1$, we derive that $F_{n+1}(x) \geq F_{n}(x)$ for all $x>0$. Hence, we conclude that $X_{n} \geq_{\text {st }} X_{n+1}$ for all $n \geq 1$. In the following theorem, we obtain the same result for a stronger stochastic order.

Theorem 3. Consider the sequence of random variables $\left\{X_{n}, n \geq 1\right\}$ as defined in (13). For all $n=1,2, \ldots$, we have

$$
X_{n} \geq_{\operatorname{lr}} X_{n+1} \text {. }
$$

Proof. From the definition of the likelihood ratio order and (17), we conclude that

$$
\frac{f_{n+1}(x)}{f_{n}(x)}=T_{n}(x), \quad n \geq 1 .
$$

The right-hand side of (19) is decreasing in $x>0$ and, hence, the claimed result follows.

Theorem 4. Let $n \geq 1$. If $X_{n}$ is ILR then

$$
X_{n} \geq_{\operatorname{lr} \uparrow} X_{n+1} .
$$

Proof. From the definition of the up-shifted likelihood ratio, it is sufficient to prove that the function $f_{n+1}(x+t) / f_{n}(x)$ is decreasing in $x$ for all $t>0$. First, from (17), we observe that

$$
\frac{f_{n+1}(x+t)}{f_{n}(x)}=T_{n}(x+t) \frac{f_{n}(x+t)}{f_{n}(x)} .
$$

On the other hand, recalling (15), we have

$$
\begin{aligned}
\frac{\partial}{\partial x}\{ & \left.T_{n}(x+t) \frac{f_{n}(x+t)}{f_{n}(x)}\right\} \\
& =\frac{\left[-\tau_{n}(x+t) f_{n}(x+t)+T_{n}(x+t) f_{n}^{\prime}(x+t)\right] f_{n}(x)-T_{n}(x+t) f_{n}(x+t) f_{n}^{\prime}(x)}{f_{n}^{2}(x)} \\
& \leq \frac{T_{n}(x+t)\left[f_{n}^{\prime}(x+t) f_{n}(x)-f_{n}(x+t) f_{n}^{\prime}(x)\right]}{f_{n}^{2}(x)}
\end{aligned}
$$


The last expression in (20) is negative since, by assumption, $X_{n}$ is ILR, i.e.

$$
\frac{f_{n}^{\prime}(x)}{f_{n}(x)} \geq \frac{f_{n}^{\prime}(x+t)}{f_{n}(x+t)} \quad \text { for all } 0<x \leq t+x .
$$

The proof is thus completed.

Remark 1. If $X$ is DRHR, i.e. $\tau(x)$ is decreasing, then $T(x)$ is convex.

Theorem 5. Let $n \geq 1$. If $X_{n}$ is DLR then $X_{n+1}$ is DLR.

Proof. From (17), we need to show that

$$
\log f_{n+1}(x)=\log f_{n}(x)+\log T_{n}(x)
$$

is convex for all $x>0$. If $X_{n}$ is DLR then $\log f_{n}(x)$ is convex. On the other hand, it is well known that if $X_{n}$ is DLR then $X_{n}$ is DRHR and, hence, $T_{n}(x)$ is convex due to Remark 1 . Since the function $\log (\cdot)$ is convex and increasing, $\log T_{n}(x)$ is convex. From (21), we have that $\log f_{n+1}(x)$ is the sum of two convex functions and then the desired result follows.

Remark 2. If $X_{n}$ is ILR then $X_{n+1}$ is not necessarily ILR. To show this fact, consider the following example.

Example 4. Suppose that $X_{1}$ has $\operatorname{CDF} F(x)=x^{\alpha}, 0<x<1(\alpha>0)$. It is easy to see that $\tau(x)=\alpha / x$ and $T(x)=-\alpha \log x, 0<x<1$. It follows that

$$
\frac{f_{1}^{\prime}(x)}{f_{1}(x)}=\frac{\alpha-1}{x}, \quad 0<x<1
$$

is decreasing in $x \in(0,1)$ for all $\alpha \geq 1$ and, hence, $X_{1}$ is ILR. (Note that $T(x)$ is convex according to Remark 1.) On the other hand, we have

$$
\frac{f_{2}^{\prime}(x)}{f_{2}(x)}=\frac{1+(\alpha-1) \log x}{x \log x}, \quad 0<x<1 .
$$

It is easy to show that the right-hand side of (22) is not decreasing in $x$ for all $\alpha \geq 1$ and, thus, $X_{2}$ is not ILR.

Proposition 5. Let $q(x)$ be a nonnegative function of $x>0$. If $q(x) \tau_{1}(x)$ is a decreasing function of $x>0$ then $q(x) \tau_{n}(x)$ is also a decreasing function of $x>0$ for all $n=1,2, \ldots$

Proof. We just show that under the hypothesis, the function $q(x) \tau_{2}(x)$ is a decreasing function of $x>0$. From (14) and (17), we have

$$
q(x) \tau_{2}(x)=q(x) \frac{f_{2}(x)}{F_{2}(x)}=q(x) \frac{T_{1}(x) f_{1}(x)}{\left(1+T_{1}(x)\right) F_{1}(x)}=q(x) \tau_{1}(x) \frac{T_{1}(x)}{1+T_{1}(x)} .
$$

Since $x /(x+1)$ is an increasing function of $x>0$ and the function $T_{1}(x)$ is decreasing with respect to $x>0$, then the function $T_{1}(x) /\left(1+T_{1}(x)\right)$ is decreasing with respect to $x>0$. From (23), we thus obtain that $q(x) \tau_{2}(x)$ is a decreasing function of $x>0$. The rest of the proof follows by induction. 
Corollary 1. If $X_{1}$ is DRHR then $X_{n}$ is DRHR for all $n \geq 2$.

Proof. The proof follows from Proposition 5 by taking $q(x)=1$ for all $x>0$.

Let us now consider the following property.

Definition 3. Let $X$ be an absolutely continuous random variable with support $\left(l_{X}, u_{X}\right)$. We say that $X$ has the DRHR in a length-biased sense (LBDRHR) if $x \tau(x)$ is decreasing in $x \in\left(l_{X}, u_{X}\right)$.

We remark that a necessary and sufficient condition such that $X$ is LBDRHR has been given in terms of stochastic comparison of quantile-based distributions in [13]. Other results on the characterization given in Definition 3 will be the subject of a future investigation.

Corollary 2. If $X_{1}$ is $L B D R H R$ then $X_{n}$ is $L B D R H R$ for all $n \geq 2$.

Proof. The proof follows from Proposition 5 by taking $q(x)=x$ for all $x>0$.

Consider now the following stochastic order from [31].

Definition 4. Let $X$ and $Y$ be absolutely continuous random variables with reversed hazard rates $\tau_{X}(x)$ and $\tau_{Y}(x)$, respectively. The random variable $X$ is said to be smaller than $Y$ in relative reversed hazard rate order (denoted by $X \leq_{\mathrm{RRH}} Y$ ), if $\tau_{Y}(x) / \tau_{X}(x)$ is an increasing function of $x$.

For instance, let $X$ and $Y$ denote the lifetimes of two components; given that the components have been found to be failed at the same time, then $X \leq_{\mathrm{RRH}} Y$ states that $Y$ has been lived longer than $X$ or, equivalently, $X$ aged faster than $Y$.

Proposition 6. The sequence of random variables defined in (13) satisfies $X_{n} \geq_{\mathrm{RRH}} X_{n+1}$ for all $n \geq 1$.

Proof. From (14), (16), and (17), we have

$$
\frac{\tau_{n+1}(x)}{\tau_{n}(x)}=\frac{f_{n+1}(x) F_{n}(x)}{F_{n+1}(x) f_{n}(x)}=\frac{T_{n}(x)}{1+T_{n}(x)} .
$$

We can see that the function $T_{n}(x) /\left(1+T_{n}(x)\right)$ is decreasing with respect to $x>0$ for all $n \geq 1$, which completes the proof.

We remark that the results stated in Theorem 3 and Proposition 6 are not related to each other, since likelihood ratio order does not imply RRH rate order and vice versa.

Theorem 6. Consider the sequence of random variables $\left\{X_{n}, n \geq 1\right\}$ as defined in (13) with the reversed hazard rate functions defined in (16), and let $Y$ be an absolutely continuous nonnegative random variable with the reversed hazard rate function $\tau_{Y}(x), x>0$. If $X_{1}$ and $Y$ satisfy the PRHRM with $G(x)=\left[F_{1}(x)\right]^{\theta}, x>0$, then $X_{n} \leq_{\mathrm{RRH}} Y$ for all $n=1,2, \ldots$

Proof. Since $\tau_{1}(x) / \tau_{Y}(x)=\theta^{-1}, x>0$, from (5), we have

$$
\frac{\tau_{2}(x)}{\tau_{Y}(x)}=\frac{f_{2}(x)}{F_{2}(x) \tau_{Y}(x)}=\frac{\tau_{1}(x)}{\tau_{Y}(x)} \frac{T_{1}(x)}{1+T_{1}(x)}=\theta^{-1} \frac{T_{1}(x)}{1+T_{1}(x)} .
$$

The function $T_{1}(x) /\left(1+T_{1}(x)\right)$ is decreasing with respect to $x>0$ and, hence, $X_{2} \leq \mathrm{RRH} Y$. The rest of the proof follows by induction. 


\section{Connection with entropy and covariance}

In this section we obtain some results about the connection between the entropy and the inactivity time of the new weighted distribution function considered in the previous section. One of the most important measures of uncertainty is the differential entropy introduced by Shannon [33]. For an absolutely continuous nonnegative random variable $X$ having PDF $f$, the differential entropy is defined by

$$
H(X)=-\int_{0}^{\infty} f(x) \log f(x) \mathrm{d} x
$$

where 'log' means natural $\log$ arithm and, by convention, $0 \log 0=0$. The entropy $H(X)$ gives the expected uncertainty contained in $f(t)$ about the predictability of an outcome of the random variable $X$. It is known that in many realistic situations, such as in survival analysis and reliability, one has information about the past time, i.e. the time elapsed after failure till time $t$, given that the unit has already failed. The entropy (24) applied to a conditioned random variable is useful in order to measure uncertainty in such situations. Di Crescenzo and Longobardi [11] indeed considered the entropy for the past lifetime, called past entropy at time $t$ of $X$, denoted by

$$
\bar{H}(t)=-\int_{0}^{t} \frac{f(x)}{F(t)} \log \frac{f(x)}{F(t)} \mathrm{d} x, \quad t>0 ;
$$

see also [28]. Furthermore, the concept of dynamic cumulative entropy as an alternative measure of uncertainty for the inactivity time was introduced in [12] and is defined as

$$
\begin{aligned}
\mathcal{C E}(X ; t) & =-\int_{0}^{t} \frac{F(x)}{F(t)} \log \frac{F(x)}{F(t)} \mathrm{d} x \\
& =-\frac{1}{F(t)} \int_{0}^{t} F(x) \log F(x) \mathrm{d} x-T(t) \tilde{\mu}(t), \quad t>0,
\end{aligned}
$$

where $\tilde{\mu}(\cdot)$ and $T(\cdot)$ are defined in (1) and (4), respectively. Note that

$$
\mathcal{C} \mathcal{E}(X)=\lim _{t \rightarrow \infty} \mathcal{C} \mathcal{E}(X ; t)=-\int_{0}^{\infty} F(x) \log F(x) \mathrm{d} x=\int_{0}^{\infty} F(x) T(x) \mathrm{d} x,
$$

where $\mathcal{C} \mathcal{E}(X)$ is called the cumulative entropy of $X$. Di Crescenzo and Longobardi [12] also showed that the dynamic cumulative entropy and the mean inactivity time are connected as

$$
\mathcal{C} \mathcal{E}(X ; t)=\mathbb{E}[\tilde{\mu}(X) \mid X \leq t], \quad t>0
$$

and, thus,

$$
\mathcal{C} E(X)=\mathbb{E}[\tilde{\mu}(X)]
$$

Now, we extend the results of [18] to the case of past time. Based on $X_{1} \stackrel{\mathrm{D}}{=} X$, we consider the sequence of random variables $\left\{X_{n}, n \geq 1\right\}$ with the corresponding distributions $F_{n}(x)$ as defined in (14), and denote by

$$
\mu_{n}(t)=\mathbb{E}\left[X_{n} \mid X_{n} \leq t\right], \quad t>0,
$$

the mean past lifetime of $X_{n}, n \geq 1$. Hereafter, we obtain the main results and connections between the dynamic cumulative entropy and the reversed hazard rate function. 
Theorem 7. For any $t>0$, and for all $n=1,2, \ldots$, we have

$$
\begin{gathered}
\mathbb{E}\left[\frac{T_{n}\left(X_{n}\right)}{\tau_{n}\left(X_{n}\right)} \mid X_{n} \leq t\right]=\mathcal{C} \&\left(X_{n} ; t\right)+\tilde{\mu}_{n}(t) T_{n}(t), \\
\operatorname{cov}\left[X_{n}, T_{n}\left(X_{n}\right) \mid X_{n} \leq t\right]=T_{n}(t)\left[\mu_{n}(t)-\mathbb{E}\left(X_{n}\right)\right]-\mathcal{C} \&\left(X_{n} ; t\right) .
\end{gathered}
$$

Proof. For $t>0$ and $n \geq 1$, we have

$$
\begin{aligned}
\mathbb{E}\left[\frac{T_{n}\left(X_{n}\right)}{\tau_{n}\left(X_{n}\right)} \mid X_{n} \leq t\right] & =\frac{1}{F_{n}(t)} \int_{0}^{t} \frac{T_{n}(x)}{\tau_{n}(x)} f_{n}(x) \mathrm{d} x \\
& =\frac{1}{F_{n}(t)} \int_{0}^{t} T_{n}(x) F_{n}(x) \mathrm{d} x \\
& =\mathcal{C} \&\left(X_{n} ; t\right)+\tilde{\mu}_{n}(t) T_{n}(t),
\end{aligned}
$$

where the last equality is obtained from (25). To prove the second expression, it is easy to show that the random variable $T_{n}\left(X_{n}\right)$ is exponentially distributed with unity mean and, hence, $\mathbb{E}\left[T_{n}\left(X_{n}\right)\right]=1$. Now, consider the following expression for $t>0$ :

$$
\begin{aligned}
\operatorname{cov}\left[X_{n}, T_{n}\left(X_{n}\right) \mid X_{n} \leq t\right]= & \mathbb{E}\left\{\left[X_{n}-\mathbb{E}\left(X_{n}\right)\right]\left[T_{n}\left(X_{n}\right)-\mathbb{E}\left(T_{n}\left(X_{n}\right)\right)\right] \mid X_{n} \leq t\right\} \\
= & \mathbb{E}\left[X_{n} T_{n}\left(X_{n}\right) \mid X_{n} \leq t\right]-\mu_{n}(t) \\
& -\mathbb{E}\left(X_{n}\right) \mathbb{E}\left[T_{n}\left(X_{n}\right) \mid X_{n} \leq t\right]+\mathbb{E}\left(X_{n}\right) .
\end{aligned}
$$

We have

$$
\begin{aligned}
\mathbb{E}\left[T_{n}\left(X_{n}\right) \mid X_{n} \leq t\right] & =\frac{1}{F_{n}(t)} \int_{0}^{t} T_{n}(x) f_{n}(x) \mathrm{d} x \\
& =\frac{1}{F_{n}(t)} \int_{0}^{t} f_{n+1}(x) \mathrm{d} x \\
& =\frac{F_{n+1}(t)}{F_{n}(t)} \\
& =1+T_{n}(t) .
\end{aligned}
$$

Also, we see that

$$
\mathbb{E}\left[X_{n} T_{n}\left(X_{n}\right) \mid X_{n} \leq t\right]=\frac{1}{F_{n}(t)} \int_{0}^{t} x f_{n+1}(x) \mathrm{d} x .
$$

Integrating by parts, we can derive

$$
\begin{aligned}
\mathbb{E}\left[X_{n} T_{n}\left(X_{n}\right) \mid X_{n} \leq t\right] & =\frac{1}{F_{n}(t)}\left[t F_{n+1}(t)-\int_{0}^{t} F_{n+1}(x) \mathrm{d} x\right] \\
& =t\left[1+T_{n}(t)\right]-\frac{1}{F_{n}(t)} \int_{0}^{t}\left[1+T_{n}(x)\right] F_{n}(x) \mathrm{d} x,
\end{aligned}
$$

and by using (25) and after simplification, we obtain

$$
\mathbb{E}\left[X_{n} T_{n}\left(X_{n}\right) \mid X_{n} \leq t\right]=\mu_{n}(t)\left[1+T_{n}(t)\right]-\mathcal{C} \mathcal{E}\left(X_{n} ; t\right) .
$$

Substituting (31) and (33) into (30), the desired result (29) finally follows. 
From Theorem 7, the following corollary is derived.

Corollary 3. Under the conditions of Theorem 7, we have, for $n \geq 1$,

$$
\begin{gathered}
\lim _{t \rightarrow \infty} \mathbb{E}\left[\frac{T_{n}\left(X_{n}\right)}{\tau_{n}\left(X_{n}\right)} \mid X_{n} \leq t\right]=\mathbb{E}\left[\frac{T_{n}\left(X_{n}\right)}{\tau_{n}\left(X_{n}\right)}\right]=\mathcal{C} \&\left(X_{n}\right), \\
\lim _{t \rightarrow \infty} \operatorname{cov}\left[X_{n}, T_{n}\left(X_{n}\right) \mid X_{n} \leq t\right]=\operatorname{cov}\left(X_{n}, T_{n}\left(X_{n}\right)\right)=-\mathcal{C} \&\left(X_{n}\right) .
\end{gathered}
$$

Remark 3. Note that the initial random variable was arbitrarily selected. Therefore, for any absolutely continuous nonnegative random variable $X$, the following identities hold:

$$
\mathbb{E}\left(\frac{T(X)}{\tau(X)}\right)=\mathcal{C} \&(X), \quad \operatorname{cov}(X, T(X))=-\mathcal{C} \&(X) .
$$

Moreover, for all $t>0$, we have

$$
\begin{gathered}
\mathbb{E}\left[\frac{T(X)}{\tau(X) \mid X \leq t}\right]=\mathcal{C} \mathcal{E}(X ; t)+\tilde{\mu}(t) T(t), \\
\operatorname{cov}[X, T(X) \mid X \leq t]=T(t)[\mu(t)-\mathbb{E}(X)]-\mathcal{C} \mathcal{E}(X ; t),
\end{gathered}
$$

where $\mu(t)=\mathbb{E}[X \mid X \leq t], t>0$, denotes the mean past lifetime of $X$.

Remark 4. Consider the sequence of random variables as defined in (13). From (14), we have, for $n \geq 1$,

$$
\bar{F}_{n+1}(x)=1-F_{n+1}(x)=\bar{F}_{n}(x)-T_{n}(x) F_{n}(x), \quad x>0 .
$$

Hence, recalling (26), we obtain the following iterative expression for the mean of $X_{n}$ :

$$
\mathbb{E}\left(X_{n+1}\right)=\mathbb{E}\left(X_{n}\right)-\mathcal{C} \mathbb{E}\left(X_{n}\right), \quad n \geq 1,
$$

which also gives a new probabilistic meaning for the cumulative entropy.

Theorem 8. For all $n=1,2, \ldots$, we have

$$
\operatorname{cov}\left(X_{n}, X_{n+1}\right)=\operatorname{cov}\left(X_{n}, X_{n}-\tilde{\mu}\left(X_{n}\right)\right)=\operatorname{var}\left(X_{n}\right)-\operatorname{cov}\left(X_{n}, \tilde{\mu}\left(X_{n}\right)\right) .
$$

Proof. From (1) and (28), we have $\mu(t)=t-\tilde{\mu}(t)$, and then

$$
\mathbb{E}\left[X_{n} X_{n+1} \mid X_{n}=t\right]=t \mathbb{E}\left[X_{n+1} \mid X_{n}=t\right]=t \mathbb{E}\left[X_{n} \mid X_{n} \leq t\right]=t\left\{t-\tilde{\mu}_{n}(t)\right\} \quad \text { for } t>0 \text {. }
$$

Hence,

$$
\mathbb{E}\left[X_{n} X_{n+1}\right]=\mathbb{E}\left[X_{n}^{2}\right]-\mathbb{E}\left[X_{n} \tilde{\mu}_{n}\left(X_{n}\right)\right] .
$$

Moreover, from (34) and (36), we have

$$
\begin{aligned}
\operatorname{cov}\left(X_{n}, X_{n+1}\right) & =\mathbb{E}\left[X_{n}^{2}\right]-\mathbb{E}\left[X_{n} \tilde{\mu}_{n}\left(X_{n}\right)\right]-\mathbb{E}^{2}\left[X_{n}\right]+\mathbb{E}\left[X_{n}\right] \mathcal{C} \mathcal{E}\left(X_{n}\right) \\
& =\operatorname{var}\left[X_{n}\right]-\operatorname{cov}\left(X_{n}, \tilde{\mu}_{n}\left(X_{n}\right)\right) \\
& =\operatorname{cov}\left(X_{n}, X_{n}-\tilde{\mu}_{n}\left(X_{n}\right)\right) .
\end{aligned}
$$

The second equality follows from $\mathcal{C} \mathcal{E}\left(X_{n}\right)=\mathbb{E}\left[\tilde{\mu}_{n}\left(X_{n}\right)\right]$, due to (27). The desired result then follows. 
Now we use the probabilistic mean value theorem (see [9]) to obtain an iterative result for $\mathcal{C} E\left(X_{n+1}\right)$. We first recall the following result (see [12, Equation (12)]).

Lemma 1. The derivative of the mean inactivity time of $X$, given in (1), can be expressed in terms of the reversed hazard rate function (when existing) as

$$
\tilde{\mu}^{\prime}(t)=1-\tau(t) \tilde{\mu}(t), \quad t>0: F(t)>0 .
$$

Theorem 9. For the sequence of random variables $\left\{X_{n}, n \geq 1\right\}$ defined in (13) and for all $n=1,2, \ldots$, we have

$$
\mathcal{C} \mathcal{E}\left(X_{n+1}\right)=\mathbb{E}\left[\tilde{\mu}_{n+1}\left(X_{n}\right)\right]-\mathbb{E}\left[\tilde{\mu}_{n+1}^{\prime}(Z)\right] \mathcal{C} \mathcal{E}\left(X_{n}\right),
$$

where $\tilde{\mu}_{n+1}^{\prime}(t)$ can be obtained from (37) and $Z$ is an absolutely continuous nonnegative random variable having $P D F$

$$
f_{Z}(z)=\frac{F_{n+1}(z)-F_{n}(z)}{\mathbb{E}\left(X_{n}\right)-\mathbb{E}\left(X_{n+1}\right)}=\frac{F_{n}(z) T_{n}(z)}{\mathcal{C} \mathcal{E}\left(X_{n}\right)}, \quad z>0 .
$$

Proof. Since $X_{n+1} \leq_{\text {st }} X_{n}$ and $\mathcal{C} \mathcal{E}\left(X_{n+1}\right)=\mathbb{E}\left[\tilde{\mu}_{n+1}\left(X_{n+1}\right)\right]$, the desired result immediately follows from [9, Proposition 3.1 and Theorem 4.1]. Note that the PDF in (39) is obtained from (14) and (34).

For the sequence of random variables defined in (13), we can verify that, for $t>0$,

$$
\tilde{\mu}_{n+1}(t)=\tilde{\mu}_{n}(t)+\frac{\mathcal{C} \mathcal{E}\left(X_{n} ; t\right)}{1+T_{n}(t)}, \quad n=1,2, \ldots
$$

Hence, from (40), we can write (38) as

$$
\mathcal{C} \mathcal{E}\left(X_{n+1}\right)=\mathcal{C} \mathcal{E}\left(X_{n}\right)\left(1-\mathbb{E}\left[\tilde{\mu}_{n+1}^{\prime}(Z)\right]\right)+\mathbb{E}\left[\frac{\mathcal{C} \mathcal{E}\left(X_{n} ; X_{n}\right)}{1+T_{n}\left(X_{n}\right)}\right], \quad n=1,2, \ldots
$$

\section{An integral operator}

Stimulated by some results shown in [18, Section 4], we now define a new operator which is dual of the $\boldsymbol{T}_{s}$ operator introduced by Dickson and Hipp [8]. It is known that the Dickson-Hipp operator for any $s \in \mathbb{R}$, denoted by $\widetilde{T}_{s} f(t)$, is defined by

$$
\boldsymbol{T}_{s} f(t)=\int_{t}^{\infty} \mathrm{e}^{-s(x-t)} f(x) \mathrm{d} x, \quad t>0
$$

where $f(x)$ is an integrable function. Applications and properties of the $\boldsymbol{T}_{s}$ operator can be found in [7], [8], and [24], among others. For simplicity, we define an operator $\widetilde{\boldsymbol{T}}_{s} f(x)$ for an integrable function $f$ and for $s \in \mathbb{R}$, by

$$
\widetilde{\boldsymbol{T}}_{s} f(t)=\int_{0}^{t} \mathrm{e}^{-s(t-x)} f(x) \mathrm{d} x, \quad t>0 .
$$

We can see that, for $t>0$, the two operators are related by the following identity:

$$
\tilde{\boldsymbol{T}}_{-s} f(t)+\boldsymbol{T}_{s} f(t)=\mathrm{e}^{s t} \mathcal{L}_{s}[f], \quad s \geq 0,
$$


where

$$
\mathcal{L}_{s}[f]=\int_{0}^{\infty} \mathrm{e}^{-s x} f(x) \mathrm{d} x, \quad s \geq 0,
$$

denotes the Laplace transform of the function $f$. Note that (41) recalls a generalized Hardy operator, similar to that considered in [5, Definition 2]. Moreover, $\boldsymbol{T}_{s} f(t)$ can be viewed as a convolution-type operator; see [1]. For instance, if $s>0$ and $f(x)$ is a PDF then, due to (41), $(1 / s) \widetilde{\boldsymbol{T}}_{s} f(t)$ is the convolution between $f(x)$ and an exponential PDF with parameter $s$.

Suppose that $X$ is an absolutely continuous nonnegative random variable with the CDF $F$. Then the $\widetilde{\boldsymbol{T}}_{s}$ operator of $F$ is defined by

$$
\widetilde{\boldsymbol{T}}_{s} F(t)=\int_{0}^{t} \mathrm{e}^{-s(t-x)} F(x) \mathrm{d} x, \quad t>0, s \in \mathbb{R} .
$$

Integrating by parts, from (41) and (42), it follows that

$$
\widetilde{\boldsymbol{T}}_{s} f(t)=F(t)-s \widetilde{\boldsymbol{T}}_{s} F(t), \quad t>0 .
$$

If $X$ is an arbitrary absolutely continuous random variable with PDF $f$ then, from (41), we have

$$
\lim _{t \rightarrow \infty} \mathrm{e}^{s t} \widetilde{\boldsymbol{T}}_{s} f(t)=\mathbb{E}\left[\mathrm{e}^{s X}\right] .
$$

Hence, (41) can be written in terms of the moment generating function of $X$ when $t$ goes to $\infty$. Now, we have an iterative result for $\widetilde{T}_{s} F_{n}(t)$.

Theorem 10. Let $\left\{X_{n}, n \geq 1\right\}$ be a sequence of absolutely continuous nonnegative random variables with the corresponding CDFs defined in (14) and let $\widetilde{\boldsymbol{T}}_{s} F_{n}(t)$ be defined as in (42). Then, for $t>0$,

$$
\widetilde{T}_{s} F_{n+1}(t)=\left[1+T_{n}(t)\right] \widetilde{T}_{s} F_{n}(t)+\widetilde{T}_{s} q_{n}(s, t), \quad n \geq 1, s \in \mathbb{R},
$$

where $q_{n}(s, t)=\tau_{n}(t) \widetilde{T}_{s} F_{n}(t)$.

Proof. From (14) and (42), we have

$$
\begin{aligned}
\widetilde{\boldsymbol{T}}_{s} F_{n+1}(t)= & \int_{0}^{t} \mathrm{e}^{-s(t-x)} F_{n}(x) \mathrm{d} x+\int_{0}^{t} \mathrm{e}^{-s(t-x)} T_{n}(x) F_{n}(x) \mathrm{d} x \\
= & \widetilde{\boldsymbol{T}}_{s} F_{n}(t)+\int_{0}^{t} \mathrm{e}^{-s(t-x)}\left[\int_{x}^{\infty} \tau_{n}(u) \mathrm{d} u\right] F_{n}(x) \mathrm{d} x \\
= & \widetilde{\boldsymbol{T}}_{s} F_{n}(t)+\int_{0}^{t} \mathrm{e}^{-s(t-x)}\left[\int_{x}^{t} \tau_{n}(u) \mathrm{d} u\right] F_{n}(x) \mathrm{d} x \\
& +\int_{0}^{t} \mathrm{e}^{-s(t-x)}\left[\int_{t}^{\infty} \tau_{n}(u) \mathrm{d} u\right] F_{n}(x) \mathrm{d} x \\
= & {\left[1+T_{n}(t)\right] \widetilde{\boldsymbol{T}}_{s} F_{n}(t)+\int_{0}^{t} \mathrm{e}^{-s(t-u)} \tau_{n}(u)\left[\int_{0}^{u} \mathrm{e}^{-s(u-x)} F_{n}(x) \mathrm{d} x\right] \mathrm{d} u } \\
= & {\left[1+T_{n}(t)\right] \widetilde{\boldsymbol{T}}_{s} F_{n}(t)+\int_{0}^{t} \mathrm{e}^{-s(t-u)} \tau_{n}(u) \widetilde{\boldsymbol{T}}_{s} F_{n}(u) \mathrm{d} u, }
\end{aligned}
$$

and the proof is completed. 
The following corollary can be obtained from (27) and (43) by setting $s=0$.

Corollary 4. Under the conditions of Theorem 10, we have, for $t>0$,

$$
\widetilde{T}_{0} F_{n+1}(t)=F_{n}(t)\left[\tilde{\mu}_{n}(t)\left(1+T_{n}(t)\right)+\mathcal{C} \mathcal{E}\left(X_{n} ; t\right)\right], \quad n \geq 1 .
$$

\subsection{Computational results}

We conclude this paper with a few illustrative examples which shed some light on the behavior of the sequences of random variables defined in (13).

Example 5. Let $X_{1}$ be uniformly distributed on [0,2]. Then we have $T_{1}(t)=-\log t / 2$, $\tilde{\mu}_{1}(t)=t / 2$, and $\mathcal{E} \mathcal{E}\left(X_{1} ; t\right)=t / 4,0<t<2$ (see [12]), so that

$$
\mathbb{E}\left(\frac{T_{1}\left(X_{1}\right)}{\tau\left(X_{1}\right) \mid X_{1} \leq t}\right)=\mathcal{C} \&\left(X_{1} ; t\right)+\tilde{\mu}_{1}(t) T_{1}(t)=\frac{t}{4}-\frac{t}{2} \log \frac{t}{2},
$$

and

$$
\operatorname{cov}\left(X_{1}, T_{1}\left(X_{1}\right) \mid X_{1} \leq t\right)=T_{1}(t)\left[\mu_{1}(t)-\mathbb{E}\left(X_{1}\right)\right]-\mathcal{C} \mathcal{E}\left(X_{1} ; t\right)=\frac{2-t}{2} \log \frac{t}{2}-\frac{t}{4} .
$$

From (35), we obtain

$$
\operatorname{cov}\left(X_{1}, X_{2}\right)=\operatorname{cov}\left(X_{1}, X_{1}-\tilde{\mu}_{1}\left(X_{1}\right)\right)=\frac{1}{2} \operatorname{var}\left(X_{1}\right)=\frac{1}{6} .
$$

Finally, from (44), we obtain

$$
\widetilde{T}_{0} F_{2}(t)=F_{1}(t)\left[\tilde{\mu}_{1}(t)\left(1+T_{1}(t)\right)+\mathcal{E}\left(X_{1} ; t\right)\right]=\frac{t^{2}}{4}\left[\frac{3}{2}-\log \frac{t}{2}\right] \text { for } 0<t<2 .
$$

It is difficult to obtain neat analytical results for the sequence of random variables $\left\{X_{n}, n \geq 1\right\}$ and, therefore, we are forced to proceed via numerical computations. To this aim, in Figure 1 we

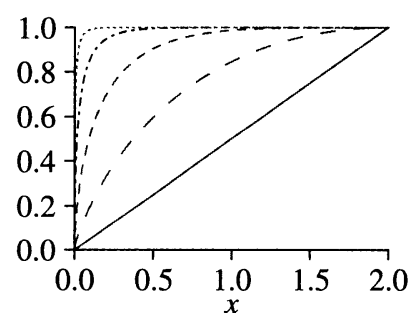

(a)

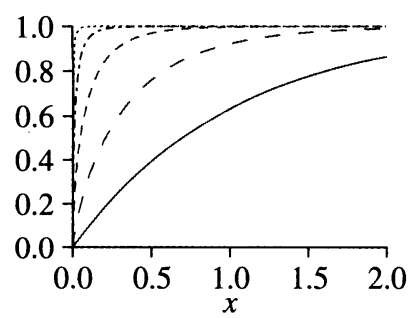

(d)

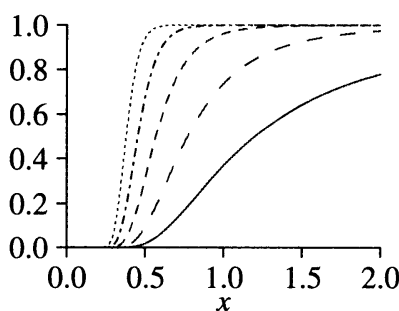

(b)

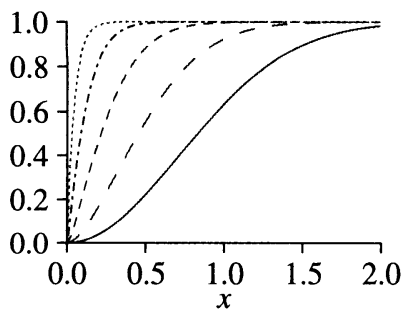

(e)

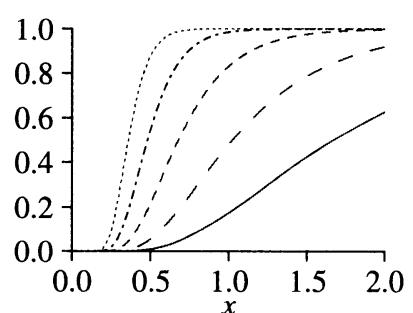

(c)

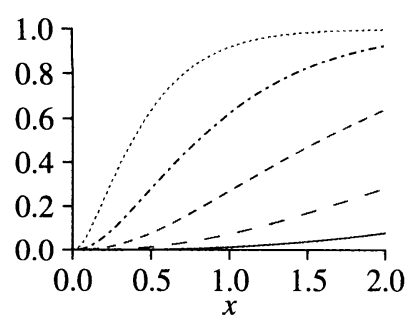

(f)

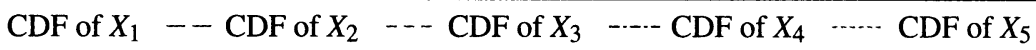

Figure 1: CDFs of $X_{1}, X_{2}, \ldots, X_{5}$ when $X_{1}$ follows the distributions given in Table 1. 
TABLE 1: Starting distribution functions.

$\begin{array}{ll}\text { (a) } & F(x)=x / 2,0<x<2 \\ \text { (b) } & F(x)=\mathrm{e}^{-x^{-2}}, x>0 \\ \text { (c) } & F(x)=\mathrm{e}^{-3 /\left(\mathrm{e}^{x}-1\right)}, x>0 \\ \text { (d) } & F(x)=1-\mathrm{e}^{-x}, x>0 \\ \text { (e) } & F(x)=1-\mathrm{e}^{-x^{2}}, x>0 \\ \text { (f) } & F(x)=1-\mathrm{e}^{-x / 2}\left(1+x / 2+x^{2} / 8\right), x>0\end{array}$

TABLE 2: The mean of the starting distribution given in Table 1.

\begin{tabular}{ccccccc}
\hline & (a) & (b) & (c) & (d) & (e) & (f) \\
\hline $\mathbb{E}\left(X_{1}\right)$ & 1.00000 & 1.77245 & 1.93791 & 1.00000 & 0.88622 & 6.00000 \\
$\mathbb{E}\left(X_{2}\right)$ & 0.50000 & 0.88662 & 1.15166 & 0.35506 & 0.50659 & 3.35935 \\
$\mathbb{E}\left(X_{3}\right)$ & 0.18066 & 0.60680 & 0.73945 & 0.10492 & 0.26035 & 1.82059 \\
$\mathbb{E}\left(X_{4}\right)$ & 0.04713 & 0.46975 & 0.51103 & 0.02483 & 0.11826 & 0.94489 \\
$\mathbb{E}\left(X_{5}\right)$ & 0.00906 & 0.38774 & 0.37544 & 0.00459 & 0.04717 & 0.46617 \\
\hline
\end{tabular}

TABLE 3: The cumulative entropy of the starting distribution given in Table 1.

\begin{tabular}{ccccccc}
\hline & $(\mathrm{a})$ & (b) & (c) & (d) & (e) & (f) \\
\hline $\mathcal{C} \mathscr{E}\left(X_{1}\right)$ & 0.50000 & 0.88623 & 0.78625 & 0.64494 & 0.37963 & 2.64065 \\
$\mathcal{C} \mathcal{E}\left(X_{2}\right)$ & 0.31934 & 0.27935 & 0.41221 & 0.25014 & 0.24624 & 1.53876 \\
$\mathcal{C} \mathcal{E}\left(X_{3}\right)$ & 0.13353 & 0.13712 & 0.22842 & 0.08009 & 0.14209 & 0.87570 \\
$\mathcal{C} \mathscr{E}\left(X_{4}\right)$ & 0.03807 & 0.08201 & 0.13559 & 0.02024 & 0.07109 & 0.47572 \\
\hline
\end{tabular}

show plots of the cumulative distribution of the random variables $X_{1}, X_{2}, \ldots, X_{5}$ for different starting distribution functions that are listed in Table 1. In the figure, the solid line corresponds to the CDF of $X_{1}$, the long-dashed line corresponds to the CDF of $X_{2}$ and so on. Moreover, we compute numerically the mean of the recursive random variables as well as the corresponding cumulative entropy; see Tables 2 and 3, respectively. Recalling (34), the cumulative entropy is computed as the difference of two consecutive means. As expected, the mean of $X_{n}$ decreases when $n$ increases, whereas the cumulative entropy decreases when $n$ increases. According to the numerical findings, we expect that the cumulative entropies are decreasing for any given starting distribution function.

\section{Acknowledgements}

We wish to thank and acknowledge partial support from the Ordered and Spatial Data Center of Excellence of Ferdowsi University of Mashhad of Iran, from GNCS-INdAM, and Regione Campania. Abdolsaeed Toomaj is grateful to the Department of Mathematics, Salerno University, for the hospitality during a four month visit in 2014.

We gratefully thank the anonymous referee for his/her careful reading of the paper and for valuable suggestions.

\section{References}

[1] Abate, J. AND Whitt, W. (1996). An operational calculus for probability distributions via Laplace transforms. Adv. Appl. Prob. 28, 75-113.

[2] Bartoszewicz, J. (2009). On a representation of weighted distributions. Statist. Prob. Lett. 79, 1690-1694. 
[3] Baxter, L. A. (1982). Reliability applications of the relevation transform. Naval Res. Logistics Quart. 29, 321-330.

[4] Block, H. W., Savits, T. H. ANd Singh, H. (1998). The reversed hazard rate function. Prob. Eng. Inf. Sci. 12, 69-90.

[5] Bloom, S. (1997). First and second order Opial inequalities. Studia Math. 126, 27-50.

[6] Burkschat, M. AND Navarro, J. (2014). Asymptotic behavior of the hazard rate in systems based on sequential order statistics. Metrika 77, 965-994.

[7] Cai, J., Feng, R. and Willmot, G. E. (2009). On the expectation of total discounted operating costs up to default and its applications. Adv. Appl. Prob. 41, 495-522.

[8] Dickson, D. C. M. AND HipP, C. (2001). On the time to ruin for Erlang(2) risk process. Insurance Math. Econom. 29, 333-344.

[9] Di Crescenzo, A. (1999). A probabilistic analogue of the mean value theorem and its applications to reliability theory. J. Appl. Prob. 36, 706-719.

[10] Di Crescenzo, A. (2000). Some results on the proportional reversed hazards model. Statist. Prob. Lett. 50, 313-321.

[11] Di Crescenzo, A. ANd Longobardi, M. (2002). Entropy-based measure of uncertainty in past lifetime distributions. J. Appl. Prob. 39, 434-440.

[12] Di Crescenzo, A. and Longobard, M. (2009). On cumulative entropies. J. Statist. Planning Inference 139, 4072-4087.

[13] Di Crescenzo, A., Martinucci, B. and Mulero, J. (2016). A quantile-based probabilistic mean value theorem. To appear in Prob. Eng. Inf. Sci.

[14] Dimitrov, B., Chukova, S. And Green, D., JR. (1997). Probability distributions in periodic random environment and their applications. SIAM J. Appl. Math. 57, 501-517.

[15] Gupta, R. C., Gupta, P. L. AND Gupta, R. D. (1998). Modeling failure time data by Lehman alternatives. Commun. Statist. Theory Meth. 27, 887-904.

[16] Gupta, R. C., Kannan, N. And Raychaudhuri, A. (1997). Analysis of lognormal survival data. Math. Biosci. 139, 103-115.

[17] Gupta, R. D. ANd Nanda, A. K. (2001). Some results on reversed hazard rate ordering. Commun. Statist. Theory Meth. 30, 2447-2457.

[18] Kapodistria, S. And Psarrakos, G. (2012). Some extensions of the residual lifetime and its connection to the cumulative residual entropy. Prob. Eng. Inf. Sci. 26, 129-146.

[19] KiJima, M. AND OHNishi, M. (1999). Stochastic orders and their application in financial optimization. Math. Meth. Operat. Res. 50, 351-372.

[20] KRAKOWSKI, M. (1973). The relevation transform and a generalization of the gamma distribution function. Rev. Française Automat. Informat. Recherche Opérat. 7, 107-120.

[21] LaU, K.-S. and Prakasa Rao, B. L. S. (1990). Characterization of the exponential distribution by the relevation transform. J. Appl. Prob. 27, 726-729.

[22] LaU, K.-S. and Prakasa RaO, B. L. S. (1992). Letter to the editor: Characterization of the exponential distribution by the relevation transform. J. Appl. Prob. 29, 1003-1004.

[23] Lehmann, E. L. (1953). The power of rank tests. Ann. Math. Statist. 24, 23-43.

[24] LI, S. AND GARRIDO, J. (2004). On ruin for the Erlang(n) risk process. Insurance Math. Econom. 34, $391-408$.

[25] LI, Y., Yu, L. AND Hu, T. (2012). Probability inequalities for weighted distributions. J. Statist. Planning Inference 142, 1272-1278.

[26] Mudholkar, G. S. and Hutson, A. D. (1996). The exponentiated Weibull family: some properties and a flood data application. Commun. Statist. Theory Meth. 25, 3059-3083.

[27] Mudholkar, G. S., Srivastava, D. K. AND Freimer, M. (1995). The exponentiated Weibull family: a reanalysis of the bus-motor-failure data. Technometrics 37, 436-445.

[28] Muliere, P., Parmigiani, G. and Polson, N. G. (1993). A note on the residual entropy function. Prob. Eng. Inf. Sci. 7, 413-420.

[29] Navarro, J., SunoJ, S. M. And Linu, M. N. (2011). Characterizations of bivariate models using dynamic Kullback-Leibler discrimination measures. Statist. Prob. Lett. 81, 1594-1598.

[30] Psarrakos, G. ANd Navarro, J. (2013). Generalized cumulative residual entropy and record values. Metrika 76, 623-640.

[31] Rezaei, M., Gholizadeh, B. and Izadkhah, S. (2015) On relative reversed hazard rate order. Commun. Statist. Theory Meth. 44, 300-308.

[32] Shaked, M. And Shanthikumar, J. G. (2007). Stochastic Orders. Springer, New York.

[33] Shannon, C. E. (1948). A mathematical theory of communication. Bell. Syst. Tech. J. 27, 379-423, $623-656$. 